\title{
Transport Vesicle Tethering at the Trans Golgi Network: Coiled Coil Proteins in Action
}

\author{
Pak-yan P. Cheung and Suzanne R. Pfeffer * \\ Department of Biochemistry, Stanford University School of Medicine, Stanford, CA, USA
}

The Golgi complex is decorated with so-called Golgin proteins that share a common feature: a large proportion of their amino acid sequences are predicted to form coiled-coil structures. The possible presence of extensive coiled coils implies that these proteins are highly elongated molecules that can extend a significant distance from the Golgi surface. This property would help them to capture or trap inbound transport vesicles and to tether Golgi mini-stacks together. This review will summarize our current understanding of coiled coil tethers that are needed for the receipt of transport vesicles at the trans Golgi network (TGN). How do long tethering proteins actually catch vesicles? Golgi-associated, coiled coil tethers contain numerous binding sites for small GTPases, SNARE proteins, and

OPEN ACCESS

Edited by:

Mary Munson,

University of Massachusetts Medical School, USA

Reviewed by:

Paul Anthony Gleeson, University of Melbourne, Australia Martin Lowe,

University of Manchester, UK

*Correspondence:

Suzanne R. Pfeffer

pfeffer@stanford.edu

Specialty section:

This article was submitted to

Membrane Traffic

a section of the journal

Frontiers in Cell and Developmental

Biology

Received: 02 January 2016

Accepted: 29 February 2016

Published: 15 March 2016

Citation:

Cheung PP and Pfeffer SR (2016) Transport Vesicle Tethering at the Trans Golgi Network: Coiled Coil Proteins in Action.

Front. Cell Dev. Biol. 4:18. doi: 10.3389/fcell.2016.00018 vesicle coat proteins. How are these interactions coordinated and are any or all of them important for the tethering process? Progress toward understanding these questions and remaining, unresolved mysteries will be discussed.

Keywords: coiled coil protein, Golgi, membrane traffic, transport vesicle, atomic force microscopy

Membrane trafficking involves the collection of cargo into transport vesicles, translocation of vesicles along cytoskeletal tracks, and tethering, docking, and fusion of vesicles at their target membranes. Tethering involves the initial capture of transport vesicle at a distance from the target membrane and the process by which the two membranes are brought together into close proximity. Tethering appears to be an important means to both enhance the efficiency and control the accuracy of subsequent, SNARE-mediated membrane fusion events (Pfeffer, 1999).

At least 15 different, long, coiled-coil-containing proteins decorate the Golgi complex and have been nicknamed, Golgins (Short et al., 2005; Munro, 2011). Given that the rise per residue in a coiled coil is typically $0.148 \mathrm{~nm}$, the majority of Golgins could theoretically extend more than $100 \mathrm{~nm}$ in length. This feature would make them well suited to connect membranes at a distance. In this review, we will discuss how a subset of long coiled coil proteins may function as tethers at the trans Golgi network (TGN) and cooperate with their binding partners to both localize to specific membrane compartments and catch inbound vesicles.

\section{TGN GOLGINS}

At the TGN in human cells, the four Golgins, Golgin-245, Golgin-97, GCC185, and GCC88, are localized to at least three distinct subdomains (Luke et al., 2003; Derby et al., 2004; Gleeson et al., 2004; Brown et al., 2011). [Because Golgin refers to a person, we prefer to refer to these proteins using an upper case: Golgin.] These dimeric Golgin proteins are present both in the cytosol and on membranes (about 50:50), and require at least one small GTPase for their TGN recruitment. Figure 1 presents their predicted content of coiled coil structure; note the significant 
sequence length differences, and the clear breaks in the predicted coiled coils, about halfway along the sequence of the two longer proteins. As described below, the N-terminus of purified GCC185 is splayed and Y-shaped (Cheung et al., 2015), a feature shared with the cis-Golgi localized, GM130 protein (Ishida et al., 2015), and likely also, with the other trans Golgi-localized Golgins shown here (because their N-termini show a lower probability of coiled-coil dimerization). The less well characterized Golgin, TMF, also has no predicted coiled coil in the first $\sim 400$ amino acids of its 1093 residue protein sequence, but significant coiled coil probability thereafter (not shown).

Golgin-245 and Golgin-97, originally identified as Golgilocalized autoantigens (Fritzler et al., 1995; Erlich et al., 1996; Griffith et al., 1997), were later found to belong to a family of proteins containing a conserved, 50-60 amino acid, C-terminal sequence called a GRIP domain (Kjer-Nielsen et al., 1999; Munro and Nichols, 1999). The GRIP domain comprises a binding site for the small GTPase, Arl1, and is important for Golgi targeting of these two Golgins (Figure 2). A conserved GRIP domain tyrosine (Y2177 in Golgin-245; Figure 2 asterisk) is essential for both Golgi targeting and Arl1 interaction (Barr, 1999; Kjer-Nielsen et al., 1999; Munro and Nichols, 1999; Lu and Hong, 2003).

Golgin-245's GRIP domain is a homodimer that interacts with two molecules of GTP-bound Arl1. The GRIP domain is comprised of three antiparallel $\alpha$ helices arranged in an Sshaped configuration (Panic et al., 2003; Wu et al., 2004); the Arl1:GRIP domain complex is anchored to the membrane via Arl1's N-terminal myristoyl group, and possibly also, a GRIP domain tryptophan residue (Panic et al., 2003; Wu et al., 2004; Lu et al., 2006). In yeast, TGN recruitment of the GRIP domaincontaining, Imh1 protein is regulated by Arl3p and the small transmembrane protein, Sys1 (Gangi Setty et al., 2003; Behnia et al., 2004).

Consistent with the importance of Arl1 binding for their TGN localizations, Golgin-245 and Golgin-97 re-localized to early endosomes when Arl1 was artificially targeted to that compartment (Derby et al., 2004). In contrast, GCC185 and GCC88 interact weakly with Arl1 (van Valkenburgh et al., 2001; Lu and Hong, 2003; Derby et al., 2004; Reddy et al., 2006) and were not relocalized under these conditions. Drosophila GCC88 but not GCC185, showed dependence on Arl1 for Golgi localization (Torres et al., 2014). Indeed, mutation of GCC185's conserved GRIP domain tyrosine had only a minimal effect on its localization (Barr, 1999; Kjer-Nielsen et al., 1999; Munro and Nichols, 1999; Lu and Hong, 2003; Derby et al., 2004; Reddy et al., 2006; Burguete et al., 2008), suggesting that another mechanism is likely in play. As described below, Rab6A facilitates Arl1 binding to GCC185's GRIP domain in vitro (Burguete et al., 2008), and can override the loss of the key tyrosine to permit Arl1 binding. In this manner, two small GTPases may cooperate to anchor GCC185 at the TGN.

Alignment of the four, human GRIP domain sequences also provides clues to their differences in Arl1 interaction (Figure 2). A lysine residue (K2196) located in the middle of Golgin-245's second GRIP domain alpha helix (Figure 2 asterisk) forms a hydrogen bond with an Arll serine residue at the binding interface (Panic et al., 2003; Wu et al., 2004). In GCC185, the equivalent residue is instead, a proline, which very likely alters the structure of the second GRIP domain alpha helix, and may explain the difference in Arl1 binding between GRIP domains. In human GCC88, a threonine replaces the lysine and may influence Arl1 binding to a lesser extent.

\section{RAB GTPASE-MEDIATED TETHER RECRUITMENT}

At least 15 different Rab GTPases may be present on the Golgi, and several interact with the TGN Golgins; Rab6A is particularly important for Golgi tether recruitment (Table 1). Rab6 recruits the p150glued subunit of the dynactin complex and the TGNlocalized, coiled coil protein, bicaudal D (Short et al., 2002). Rab6 interacts with bicaudal D's C-terminal coiled coil (Matanis et al., 2002); coordination between bicaudal D1/D2, Rab6 and the dynein-dynactin complex targets bicaudal D1/D2 to the Golgi (Short et al., 2002). The yeast protein, Sgm1 is recruited to the Golgi by interacting with the Rab6 ortholog, Ypt6 (Siniossoglou and Pelham, 2001). Sgml's mammalian homolog, TMF, also interacts with three mammalian orthologs of Ypt6: Rab6A, Rab6A', and Rab6B, via the C-terminal coiled coil domain, for Golgi localization (Fridmann-Sirkis et al., 2004; Siniossoglou, 2005; Yamane et al., 2007). Moreover, as described below, the kinesin-family motor, KIF1C can act as a tether under certain circumstances and is recruited to the Golgi via C-terminal, Rab6A binding (Lee et al., 2015).

Rab6A binds GCC185 just upstream of the C-terminal GRIP domain, which facilitates Arl1 binding to this GRIP domain in vitro (Burguete et al., 2008). A stable attachment platform supporting GCC185 on the TGN is formed by anchoring two Rab6 molecules to the TGN membrane with the four Cterminal geranylgeranyl groups accommodated by an extended, Rab GTPase hypervariable domain; insertion of Arl1 myristoyl groups into the membrane (Panic et al., 2003; Wu et al., 2004) and possibly the membrane insertion of GCC185's C-terminus (Panic et al., 2003) would further stabilize this interaction. This model explains the inability of Arl1 alone to mediate GCC185's Golgi localization.

Houghton et al. (2009) challenged the roles of Arl1 and Rab6 in GCC185 localization. In that study, those workers used siRNA to achieve $80 \%$ depletion of Rab6; under those conditions, they failed to alter the Golgi association of GCC185. Burguete et al. (2008) required at least 90\% depletion of Rab6 to alter GCC185 localization, which readily explains this discrepancy. GCC185 also binds CLASP proteins and other Rab GTPases, all of which may contribute to the Golgi localization of this Golgin. Nevertheless, the only fragment of GCC185 that is sufficient for Golgi localization is the C-terminal region comprised of the GRIP and Rab6 binding domains (Burguete et al., 2008; Hayes et al., 2009). Given the overall, structural conservation of GRIP domain Golgins, it seems unlikely that GCC185 would be very functionally distinct from other TGN-localized, GRIP domain proteins, despite the fact that its Golgi localization mechanism may be somewhat more complex. Moreover, recent work (Bardin 


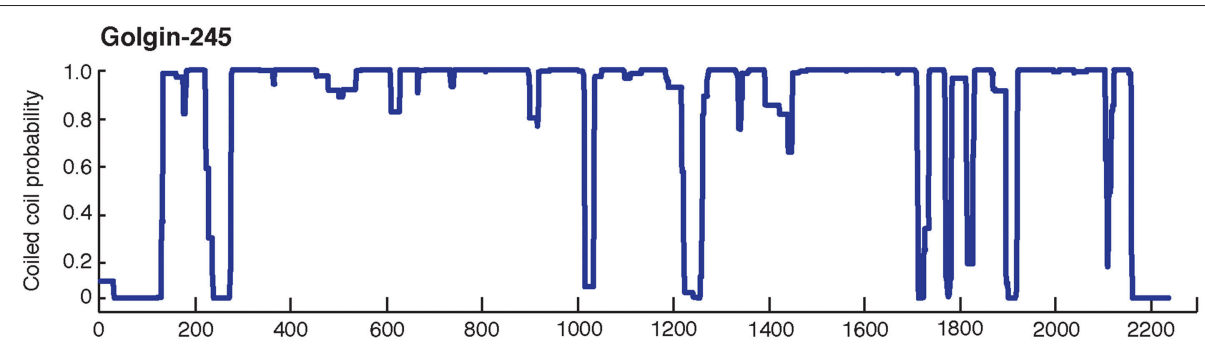

GCC185

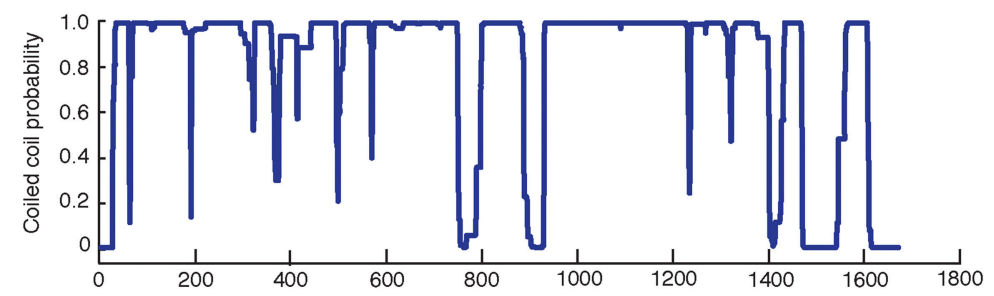

GCC88

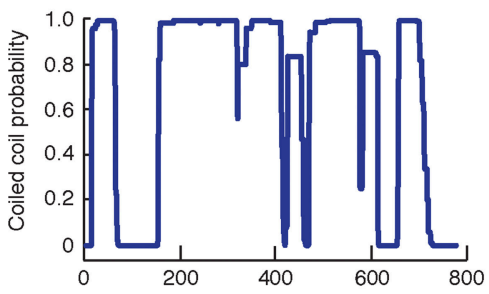

Golgin-97

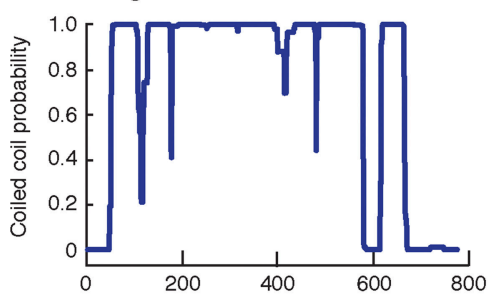

FIGURE 1 | Predicted probability of each amino acid in the sequences of the four TGN Golgins to form a coiled-coil structure. Top to bottom: Golgin-245, GCC185, GCC88, and Golgin-97. The central coiled coil region and the adjacent breaks of GCC185 and Golgin-245 likely form a central bubble.

et al., 2015) supports a model in which Rab6 may cooperate with Arl1 (and perhaps additional factors) for mammalian GCC185 Golgi localization. Note that targeting of Drosophila GCC185 to the TGN is not affected by the absence of either or both Arl1 and Rab6 (Torres et al., 2014), but the C-terminal half of the Drosophila protein (residues 582-1127) is only 26\% identical to the corresponding region of the 1684 residue human GCC185 protein (46\% similar with major gaps).

\section{HOW DO TETHERS CATCH VESICLES?}

Little is known about how long, coiled coil Golgins actually capture transport vesicles. Their roles as tethers have been inferred by the phenotypes seen upon their depletion from cultured cells. Golgi fragmentation is always detected; in some cases, transport vesicle intermediates also accumulate in the cytoplasm. Thus, long tethers can have dual roles, and work on GCC185 has shown that Golgi ribbon structure stabilization and transport vesicle tethering can utilize different regions of this protein (Brown et al., 2011).

By definition, tethering proteins should have the capacity to bind transport vesicles directly, to link them to the corresponding target membranes. Yet to date, few TGN tethers have been clearly shown to do this. Reflecting tethering events that take place earlier in the Golgi, Warren and colleagues used purified CASP and 115 proteins to capture distinct classes of transport vesicles enriched in Golgin-84 or p24 proteins (Malsam et al., 2005). However, the precise domains of CASP and p115 responsible for vesicle binding is still not known, and how or whether the proteins bring these vesicles closer to their corresponding target membranes is also unknown.

The notion that tethering proteins use their N-termini to "catch" vesicles, while being anchored at target membranes via their C-termini, was reinforced upon characterization of GMAP210. This cis-Golgi tether has a curvature-sensing, amphipathic lipid-packing sensor (ALPS) motif at its extreme N-terminus 


\begin{tabular}{|c|c|c|}
\hline golgin-245 & & 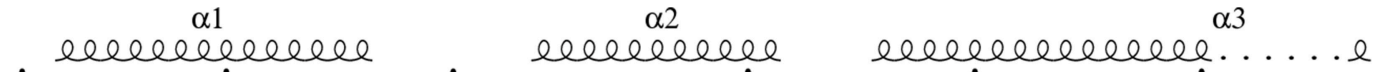 \\
\hline $\begin{array}{l}\text { golgin-245 } \\
\text { golgin-97 } \\
\text { GCC88 } \\
\text { GCC185 }\end{array}$ & $\begin{array}{l}1 \\
1 \\
1 \\
1\end{array}$ & 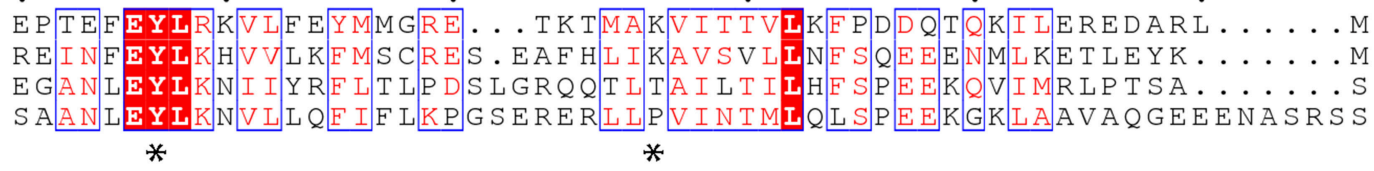 \\
\hline golgin-245 & & ele..... \\
\hline $\begin{array}{l}\text { golgin-245 } \\
\text { golgin-97 } \\
\text { GCC88 } \\
\text { GCC185 }\end{array}$ & $\begin{array}{l}52 \\
53 \\
54 \\
61\end{array}$ & 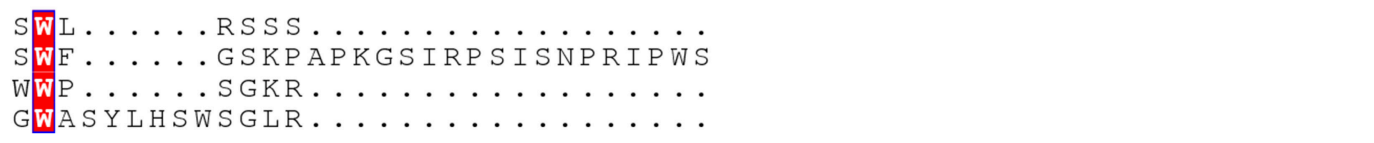 \\
\hline \multicolumn{3}{|c|}{$\begin{array}{l}\text { FIGURE } 2 \text { | Sequence alignment of the GRIP domains of human Golgin-245, Golgin-97, GCC88, and GCC185 based on the crystal structure of } \\
\text { Golgin-245 generated from PROMALS3D (Pei et al., 2008) and ESPript 3.0 (Robert and Gouet, 2014). The secondary structures of human Golgin-245 are } \\
\text { shown above. Invariant residues are in white with red background; similar residues (with global scores between } 0.7 \text { and 1.0) are in red, framed in blue, and other } \\
\text { residues are in black. Asterisks indicate the invariant tyrosine that is critical for Golgin-245 and Golgin-97 localization and Arl1 binding, as well as the proline in } \\
\text { GCC185 that may break helix } 2 \text {. }\end{array}$} \\
\hline
\end{tabular}

that binds liposomes of high membrane curvature (radius < $50 \mathrm{~nm}$ ) but not flat membranes in vitro (Drin et al., 2007, 2008). Upon heterologous expression in yeast cells (that normally lack GMAP-210), this ALPS motif was targeted to uncoated vesicles carrying markers from the ER-Golgi interface and late Golgi (Pranke et al., 2011), which likely reflects their small size and/or specific lipid compositions. In mammalian cells, GMAP-210's N-terminal ALPS domain was recently shown to be sufficient to tether vesicles upon relocation to mitochondria (Sato et al., 2015; see also Wong and Munro, 2014). Such relocation did not require Rab2 binding sites located in the central coiled coil region of GMAP-210; these sites were, however, essential for GMAP210 's ability to maintain normal Golgi structure. From this, Lowe and colleagues concluded that Rab2's role is downstream of Nterminal, ALPS motif-mediated vesicle binding (Sato et al., 2015), and again separable from vesicle capture.

GMAP-210 is the only Golgin that contains an ALPS motif, thus other Golgins must tether vesicles by another mechanism. Several Golgi tethers interact with vesicle coat proteins (Cai et al., 2007; Bröcker et al., 2010). For example, cis-Golgi localized p115 can interact directly with $\beta$ COP to facilitate vesicle tethering (Guo et al., 2008). The interaction maps to p115's N-terminal, globular domain and $\beta$ COP's so-called FW motif. Thus, p115 uses its $\mathrm{N}$-terminal heads to bind the vesicle coat and its coiled coil domain to bind to Rab1 (Beard et al., 2005), syntaxin-5, and GOS-28 on target membranes (Shorter et al., 2002; Guo et al., 2008).

GCC185 can bind the AP-1 clathrin adaptor that likely decorates the vesicles it captures (Brown et al., 2011; Table 1). AP-1 interacts with the cytoplasmic domains of mannose 6-phosphate receptors [(MPRs); Glickman et al., 1989] and functions in the both the export of MPRs from the TGN to endosomes as well as their transport from late endosomes back to the TGN (Meyer et al., 2000; Braulke and Bonifacino, 2009). GCC185's AP-1 binding site near the middle of this tether (just beyond the central bubble, see below), is required for the overall process of MPR-vesicle tethering (Brown et al., 2011).

Very recently, GCC185's N-terminus was also shown to be capable of binding transport vesicles directly, in vitro (Cheung et al., 2015). This is consistent with the essential nature of the $\mathrm{N}$-terminal portion for GCC185's roles in both Golgi ribbon maintenance and vesicle capture (Hayes et al., 2009). Using atomic force microscopy, purified GCC185 was shown to occur as a parallel dimer of $\sim 145 \mathrm{~nm}$ in length (Figure 3). The $\mathrm{N}$ terminus of most molecules was splayed, and a splayed $\mathrm{N}$ terminus was shown to be most effective in binding transport vesicles in vitro.

Given that GCC185 contains an AP-1 binding site just Cterminal to the bubble shown in Figure 3, the vesicle may bind first to the N-terminal "arms" which might then transfer the vesicle to the AP-1 site; alternatively, the vesicle could sit on the AP-1 site and bubble, and be "hugged" by the flexible, N-terminal arms. Moreover, a Rab9 binding site is located near the bubble, but this has been shown to be dispensible for GCC185 function (Brown et al., 2011); perhaps there is additional Rab9-binding site closer to the N-terminus, as indicated in Figure 3. Obviously, additional work is needed to better define the events responsible for vesicle delivery to the TGN.

Another surprise from this study was the finding that GCC185 is significantly shorter than predicted by coiled coil prediction algorithms; moreover, the dimeric coiled coil was unwound about halfway along the length of the protein, creating an internal bubble and making the full length protein appear rather floppy, overall (Figure 3). Deletion of specific, bubble-forming sequences made GCC185 less likely to bend and importantly, incapable of rescuing transport vesicle receipt at the TGN. In addition, the central bubble could be replaced by unstructured, gly-ser repeats in vesicle tethering-rescue experiments, showing that these sequences provide flexibility rather than a partner protein-binding site. These experiments show that GCC185 can 
TABLE 1 | TGN Golgins and tether-like proteins and binding partners.

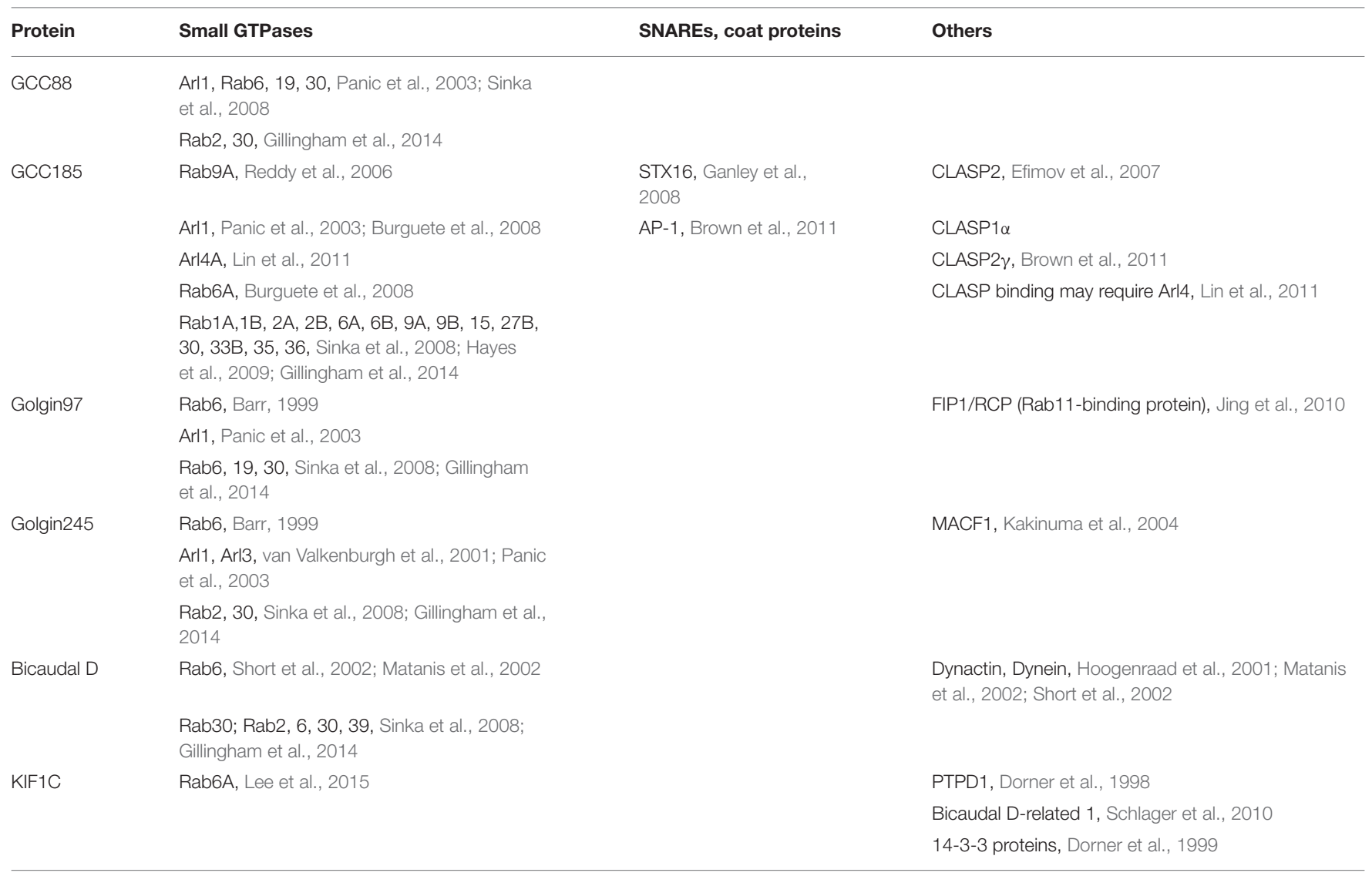

bind vesicles at its N-terminus, and also elsewhere along its length, either simultaneously or as part of a multistep process. Vesicle binding by a Y-shaped N-terminus provides avidity for the binding reaction. Importantly, TGN tethers appear to be much more flexible than originally anticipated, capable of collapse onto the Golgi surface to bring vesicles into proximity of the membrane (Cheung et al., 2015; Figure 3).

Wong and Munro (2014) recently used rapamycin-controlled, hetero-dimerization domains to induce relocalization of Golgins to the surface of mitochondria. They found that within only $15 \mathrm{~min}$ of rapamycin treatment, membranes containing MPRs and the Vtila SNARE protein relocalize to mitochondria decorated with any one of three different TGN Golgins: Golgin245, Golgin-97, and GCC88. Since the Golgins are known to be important for Golgi ribbon maintenance, it was not fully established whether this particular tethering represents trapping of Golgi membranes rather than actual retrograde transport intermediates (especially since Vtila is a TGN-localized tSNARE). This is an important distinction because only a tiny proportion of the markers evaluated are present in transport carriers at steady state, yet a large proportion of those marker proteins re-localized to mitochondria in a very short time frame (15 min). If stacks are being relocalized, it would indicate that the TGN tethers studied are most potent in cisternal linking, rather than actual transport intermediate capture. This would easily explain the lack of activity observed for GCC185, a Golgin that is well established to be required for MPR and Shiga toxin retrograde transport in cells (Reddy et al., 2006; Derby et al., 2007; Hayes et al., 2009; Brown et al., 2011) and now shown to bind MPR- and Rab9A-containing vesicles in vitro (Cheung et al., 2015).

Wong and Munro (2014) included an anti-CD8 antibody uptake experiment, in conjunction with a CD8-MPR chimera, to be sure that the cargo monitored represented transport from the endocytic pathway; they concluded that Golgin-97, but not GCC185 localizes this construct to mitochondria after $15 \mathrm{~min}$. It takes a CD8-MPR chimeric protein at least $30-45 \mathrm{~min}$ to reach the Golgi from the cell surface under normal conditions. In addition, Golgin-97 appears to be a weak tether (if a tether at all) for normal MPR retrograde transport in cells, upon siRNA depletion, as monitored by TGN-specific, tyrosine sulfation of the cation-dependent MPR (Reddy et al., 2006). It will be important to show that Golgin-97 is actually needed for CD8MPR recycling, which is likely to represent early endosome to Golgi transport rather than late endosome to Golgi transport taken by the native MPR. Nevertheless, Wong and Munro (2014) obtained very beautiful vesicle tethering data for tethers that localize to earlier Golgi compartments.

Are TGN Golgins promiscuous (Wong and Munro, 2014) and do they overlap in function? While this may certainly be true for their Golgi ribbon maintenance activities, TGN Golgins have been shown to differ in their abilities to support distinct 


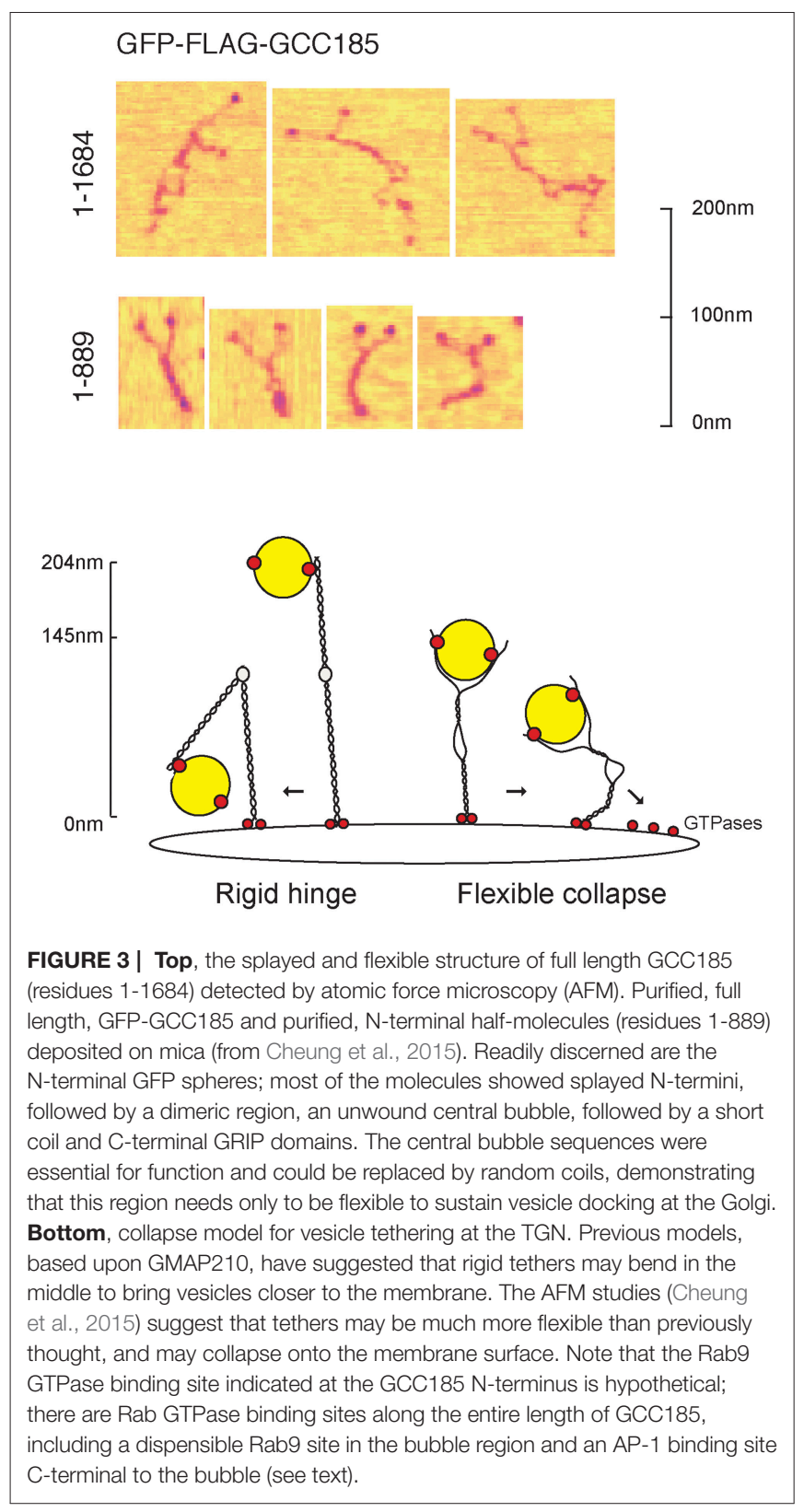

retrograde transport pathways. Derby et al. (2007) showed that Shiga toxin, but not TGN38, requires GCC185 for retrograde transport, while TGN38 and MPRs seem to rely on GCC88 (Lieu and Gleeson, 2010). As mentioned above, Reddy et al. (2006) showed that MPR transport relies on GCC185 and much less so, upon Golgin-97. Moreover, depletion of any one of several (but not all), individual Golgins is sufficient to fragment the Golgi into mini-stacks, despite the presence of the other Golgins (Diao et al., 2003; Fridmann-Sirkis et al., 2004; Yoshino et al., 2005; Zolov and Lupashin, 2005; Puthenveedu et al., 2006; Feinstein and Linstedt, 2008). These data suggest that the TGN Golgins are not functionally redundant for transport vesicle tethering.

Golgins contain multiple Rab GTPase binding sites along their lengths (Diao et al., 2003; Beard et al., 2005; Sinka et al., 2008; Hayes et al., 2009; Gillingham et al., 2014). Many of these binding sites are located within regions that are not needed for Golgi localization, implying that Rab:Golgin interactions are involved in vesicle tethering and ribbon maintenance, in addition to Golgi localization. Of the 14 different Rabs that can bind to human GCC185 in vitro (Hayes et al., 2009), most are Golgi-localized, but Rab9A is present on inbound transport vesicles (Barbero et al., 2002) and can also bind GCC185. These data imply that vesicle associated Rabs contribute to the vesicle tethering process.

Vesicle-associated Rab proteins can also interact with tethers via linker proteins. The Rab11 effector protein, FIP1/RCP, interacts with Golgin-97 and is required for the retrograde transport of TGN38 (Jing et al., 2010). Golgin-97 binds to the C-terminus of FIP/RCP at a region just upstream of the Rab11binding domain, suggesting that FIP1/RCP may act like a linker to bring Rab11-containing membranes close to Golgin-97 to mediate the docking of a retrograde carrier.

\section{LENGTH AND SHAPE MATTER}

The length of a tether determines how far it is able to reach out into the cytoplasm. Electron microscopic studies of p115 and its yeast homolog Uso1 showed extended, rod-like structures of their dimeric, carboxy-terminal coiled coil domains (Sapperstein et al., 1995; Yamakawa et al., 1996). The measured lengths of the rod domains of the two proteins are in good accordance with their predicted lengths.

GMAP210 length is important for its function: a miniGMAP construct containing an intact $\mathrm{N}$-terminal vesicle binding domain, central Rab2 binding domain, and C-terminal GRAB domain cannot rescue Golgi ribbon morphology (Sato et al., 2015). Although apparent tethering activity was not affected when mini-GMAP210 was targeted to mitochondria (Wong and Munro, 2014), the inability to function properly at the Golgi implies that the length of this tether is important for its native function on the Golgi surface. Sato et al. (2015) hypothesize that this includes reaching out through a meshwork of multiple Golgi tethers. It may also be due to the loss of a specific sequence that is important for normal Golgi ribbon maintenance.

GCC185 has been shown to play independent roles in Golgi structure maintenance and transport vesicle tethering (Hayes et al., 2009; Brown et al., 2011). Functional rescue experiments showed that GCC185 does not have to be especially long to support Golgi structure (less than $50 \%$ of full length), but a construct of $\sim 60 \%$ of its full length, including the first coiled coil domain, is absolutely required for MPR vesicle tethering (Hayes et al., 2009; Brown et al., 2011). Importantly, the full length protein appears to be $\sim 145 \mathrm{~nm}$ in length (Cheung et al., 2015), $71 \%$ shorter than expected for a protein with such high coiledcoil propensity. Thus, tethering may occur on a shorter distance scale than originally anticipated.

\section{GOLGI RIBBON MAINTENANCE-CLUES FROM UNEXPECTED, NOVEL TETHERS}

How do TGN tethers contribute to ribbon maintenance? As mentioned earlier, they contain Golgi-localized, Rab GTPase binding sites along their lengths, and may also interact with 
additional binding partners that enable them to link regions of the Golgi together. GCC185 N-termini contain Rab and Arl4 binding sites (Hayes et al., 2009; Brown et al., 2011; Lin et al., 2011) and CLASP binding sites for microtubule association (Matsui et al., 2015). Interestingly, GCC185 residues 1331-1573, when removed from the rest of the molecule, render the protein unable to rescue Golgi ribbon morphology. This suggests that the role of this protein in ribbon maintenance must involve a specific, protein:protein interaction that is essential for this aspect of its function and distinct from interactions that drive vesicle tethering. That a region is needed for ribbon maintenance but not for vesicle capture indicates that these processes are also, molecularly distinct.

Support for simple, end-to-end binding reactions as a model to explain tether maintenance of Golgi ribbon structure comes from an unexpected source. KIF1C is an anterograde, kinesin family motor with an $\mathrm{N}$-terminal motor domain. The protein participates in cargo transport from the TGN to the cell surface, and it is also needed to maintain an intact Golgi ribbon structure. Recently, Lee et al. (2015) reported that KIF1C can bind Rab6A at its C-terminus to link this motor to its membrane-bound cargo. More surprising was the observation that KIF1C, but not the related KIF1A protein, can also bind Rab6A via its $\mathrm{N}$-terminal motor domain, and motor domain binding interferes with the ability of the KIF1C motor to bind to microtubules, decreasing the affinity for microtubules by at least 10 fold. KIF1C's ability to bind Rab6A at each end correlated with its ability to maintain an intact Golgi ribbon, even upon nocodazole treatment to disrupt microtubule tracks. In addition, a mutant KIF1C lacking motor activity still had the capacity to maintain Golgi ribbon structure if $\mathrm{N}$ - and C-terminal Rab6A binding sites were intact (Lee et al., 2015). These findings suggest that non-Golgin proteins can also function as membrane tethers, and may help transport vesicles hold on to their target membranes to enhance the probability of membrane fusion. The presence of Rab GTPases binding sites at both ends of KIF1C was all that was required to hold the ribbon together. Also, note that Rab6A is on vesicles surrounding and leaving the Golgi. Thus, a molecule of KIF1C anchored on a vesicle via its C-terminal, Rab6A binding domain can be tethered to a Rab6A-containing Golgi domain via the N-terminal motor. As Rab6A becomes less abundant, the motor could instead hop onto a microtubule track and move away from the Golgi complex.

\section{AFTER BINDING-WHAT NEXT?}

Tethers bind vesicles at a distance, and this interaction is presumed to increase the probability of engagement between vesicle and target SNARE proteins (Pfeffer, 1999). Aside from increasing the chances of target membrane engagement, do tethers do more? The "tentacular meshwork" model (Sinka et al., 2008; Munro, 2011) proposes that tethers act collectively by forming a meshwork surrounding the Golgi. According to this model, Rab binding sites on multiple Golgins entrap vesicles as they undergo multiple rounds of association and dissociation, moving along or between Golgins. Slowing vesicle diffusion will increase the probability of membrane target encounters. This is similar to a "hopping" model in which vesicles hop from one Rab binding site to the next, along a Golgin tether (Hayes et al., 2009; Ramirez and Lowe, 2009), or the "vesicle on a string" concept derived from the fibrous elements detected by electron microscopy connecting in vitro-generated COP-I vesicles to Golgi cisternae (Melancon et al., 1987; Weidman et al., 1993; Simon et al., 1996; Orci et al., 1998). Some version of this model is likely to be correct, even if Golgins don't protrude from the surface of the Golgi complex as static, rigid rods. Most Golgin tethers contain breaks between predicted, coiled coil domains that likely represent highly flexible hinge regions (Figure 1). Indeed, the collapsible nature of the purified, GCC185 tether (Figure 3) suggests an alternative structure for the Golgin meshwork that may be shorter and less rigid, but could nevertheless, slow vesicle diffusion.

Finally, some tethering proteins are also able to catalyze SNARE complex assembly. The first indication of such a role came from a study of the early Golgi protein, p115 (Shorter et al., 2002; see also Puthenveedu and Linstedt, 2004). GCC185 binds syntaxin-16 and the interaction can be competed by Rab6A (Ganley et al., 2008); GM130 interacts with Syntaxin5 , and p115 interferes with this interaction (Diao et al., 2008), but the functional consequences of these interactions remain to be determined (Table 1). Much remains to be discovered regarding how long coiled coil tethers might regulate SNARE complex assembly; their SNARE binding capacity may also be equally important to assemble a fusion-competent membrane immediately adjacent to the membrane-anchored, vesicle tether.

\section{SUMMARY AND FUTURE PERSPECTIVES}

TGN tethers participate in both Golgi ribbon maintenance and vesicle tethering, and at least for GCC185 and GMAP-210, can do so via distinct and independent domains. These proteins are likely not as long as originally presumed, and structural analysis of each of them will reveal additional clues to their mechanisms of action. Splayed N-termini may represent a more common feature than originally anticipated, providing avidity to tethering reactions. Finally, possible tether collapse onto the Golgi surface will now be an important new mechanism to test more directly, using whatever approaches are available, to provide clues to this interesting, possible new mechanism for vesicle tethering on the Golgi. How do tethers release their cargoes? Why don't cytosolic pools of tethers interfere with the tethering process? Why does the Golgi ribbon fragment so readily? Clearly there remains a great deal yet to discover.

\section{AUTHOR CONTRIBUTIONS}

All authors listed, have made substantial, direct and intellectual contribution to the work, and approved it for publication.

\section{FUNDING}

This research was funded by a grant from the NIH (DK37332). 


\section{REFERENCES}

Barbero, P., Bittova, L., and Pfeffer, S. R. (2002). Visualization of Rab9- mediated vesicle transport from endosomes to the trans-Golgi in living cells. J. Cell Biol. 156, 511-518. doi: 10.1083/jcb.200109030

Bardin, S., Miserey-Lenkei, S., Hurbain, I., Garcia-Castillo, D., Raposo, G., and Goud, B. (2015). Phenotypic characterisation of RAB6A knockout mouse embryonic fibroblasts. Biol. Cell. 107, 427-439. doi: 10.1111/boc.2014 00083

Barr, F. A. (1999). A novel Rab6-interacting domain defines a family of Golgitargeted coiled-coil proteins. Curr. Biol. 9, 381-384. doi: 10.1016/S09609822(99)80167-5

Beard, M., Satoh, A., Shorter, J., and Warren, G. (2005). A cryptic rab1-binding site in the p115 tethering protein. J. Biol. Chem. 280, 25840-25848. doi: 10.1074/jbc.M503925200

Behnia, R., Panic, B., Whyte, J. R., and Munro, S. (2004). Targeting of the Arf-like GTPase Arl3p to the Golgi requires N-terminal acetylation and the membrane protein Syslp. Nat. Cell Biol. 6, 405-413. doi: 10.1038/ncb1120

Braulke, T., and Bonifacino, J. S. (2009). Sorting of lysosomal proteins. Biochim. Biophys. Acta 1793, 605-614. doi: 10.1016/j.bbamcr.2008.10.016

Bröcker, C., Engelbrecht-Vandré, S., and Ungermann, C. (2010). Multisubunit tethering complexes and their role in membrane fusion. Curr. Biol. 20, 943-952. doi: 10.1016/j.cub.2010.09.015

Brown, F. C., Schindelhaim, C. H., and Pfeffer, S. R. (2011). GCC185 plays independent roles in Golgi structure maintenance and AP-1-mediated vesicle tethering. J. Cell Biol. 194, 779-787. doi: 10.1083/jcb.201104019

Burguete, A. S., Fenn, T. D., Brunger, A. T., and Pfeffer, S. R. (2008). Rab and Arl GTPase family members cooperate in the localization of the Golgin GCC185. Cell 132, 286-298. doi: 10.1016/j.cell.2007.11.048

Cai, H., Yu, S., Menon, S., Cai, Y., Lazarova, D., Fu, C., et al. (2007). TRAPPI tethers COPII vesicles by binding the coat subunit Sec23. Nature 445, 941-944. doi: 10.1038/nature05527

Cheung, P. P., Limouse, C., Mabuchi, H., and Pfeffer, S. R. (2015). Protein flexibility is required for vesicle tethering at the Golgi. eLife 4:e12790. doi: 10.7554/eLife. 12790

Derby, M. C., Lieu, Z. Z., Brown, D., Stow, J. L., Goud, B., and Gleeson, P. A. (2007). The trans-Golgi network Golgin, GCC185, is required for endosometo-golgi transport and maintenance of golgi structure. Traffic 8, 758-773. doi: 10.1111/j.1600-0854.2007.00563.x

Derby, M. C., van Vliet, C., Brown, D., Luke, M. R., Lu, L., Hong, W., et al. (2004). Mammalian GRIP domain proteins differ in their membrane binding properties and are recruited to distinct domains of the TGN. J. Cell Sci. 117, 5865-5874. doi: $10.1242 /$ jcs. 01497

Diao, A., Frost, L., Morohashi, Y., and Lowe, M. (2008). Coordination of Golgin tethering and SNARE assembly: GM130 binds syntaxin 5 in a p115regulated manner. J. Biol. Chem. 283, 6957-6967. doi: 10.1074/jbc.M7084 01200

Diao, A., Rahman, D., Pappin, D. J., Lucocq, J., and Lowe, M. (2003). The coiled-coil membrane protein Golgin-84 is a novel rab effector required for Golgi ribbon formation. J. Cell Biol. 160, 201-212. doi: 10.1083/jcb.2002 07045

Dorner, C., Ciossek, T., Müller, S., Møller, P. H., Ullrich, A., and Lammers, R. (1998). Characterization of KIF1C, a new kinesin-like protein involved in vesicle transport from the Golgi apparatus to the endoplasmic reticulum. J. Biol. Chem. 273, 20267-20275. doi: 10.1074/jbc.273.32.20267

Dorner, C., Ullrich, A., Häring, H. U., and Lammers, R. (1999). The kinesinlike motor protein KIF1C occurs in intact cells as a dimer and associates with proteins of the 14-3-3 family. J. Biol. Chem. 274, 33654-33660. doi: $10.1074 / \mathrm{jbc} .274 .47 .33654$

Drin, G., Casella, J. F., Gautier, R., Boehmer, T., Schwartz, T. U., and Antonny, B. (2007). A general amphipathic alpha-helical motif for sensing membrane curvature. Nat. Struct. Mol. Biol. 14, 138-146. doi: 10.1038/nsmb1194

Drin, G., Morello, V., Casella, J. F., Gounon, P., and Antonny, B. (2008). Asymmetric tethering of flat and curved lipid membranes by a Golgin. Science 320, 670-673. doi: 10.1126/science.1155821

Efimov, A., Kharitonov, A., Efimova, N., Loncarek, J., Miller, P. M., Andreyeva, N., et al. (2007). Asymmetric CLASP-dependent nucleation of noncentrosomal microtubules at the trans-Golgi network. Dev. Cell. 12, 917-930. doi: 10.1016/j.devcel.2007.04.002

Erlich, R., Gleeson, P. A., Campbell, P., Dietzsch, E., and Toh, B. H. (1996). Molecular characterization of trans-Golgi p230. A human peripheral membrane protein encoded by a gene on chromosome 6 p12-22 contains extensive coiled-coil alpha-helical domains and a granin motif. J Biol.Chem. 271, 8328-8337.

Feinstein, T. N., and Linstedt, A. D. (2008). GRASP55 regulates Golgi ribbon formation. Mol. Biol. Cell 19, 2696-2707. doi: 10.1091/mbc.E07$11-1200$

Fridmann-Sirkis, Y., Siniossoglou, S., and Pelham, H. R. B. (2004). TMF is a Golgin that binds Rab6 and influences Golgi morphology. BMC Cell Biol. 5:18. doi: $10.1186 / 1471-2121-5-18$

Fritzler, M. J., Lung, C. C., Hamel, J. C., Griffith, K. J., and Chan, E. K. L. (1995). Molecular characterization of Golgin-245, a novel Golgi complex protein containing a granin signature. J. Biol. Chem. 270, 31262-31268. doi: $10.1074 /$ jbc.270.52.31262

Gangi Setty, S. R., Shin, M. E., Yoshino, A., Marks, M. S., and Burd, C. G. (2003). Golgi recruitment of GRIP domain proteins by Arf-like GTPase 1 is regulated by Arf-like GTPase 3. Curr. Biol. 13, 401-404. doi: 10.1016/S09609822(03)00089-7

Ganley, I. G., Espinosa, E., and Pfeffer, S. R. (2008). A syntaxin 10-SNARE complex distinguishes two distinct transport routes from endosomes to the trans-Golgi in human cells. J. Cell Biol. 180, 159-172. doi: 10.1083/jcb.2007 07136

Gillingham, A. K., Sinka, R., Torres, I. L., Lilley, K. S., and Munro, S. (2014). Toward a comprehensive map of the effectors of rab GTPases. Dev. Cell. 31, 358-373. doi: 10.1016/j.devcel.2014.10.007

Gleeson, P. A., Lock, J. G., Luke, M. R., and Stow, J. L. (2004). Domains of the TGN: coats, tethers and G proteins. Traffic 5, 315-326. doi: 10.1111/j.13989219.2004.00182.x

Glickman, J. N., Conibear, E., and Pearse, B. M. F. (1989). Specificity of binding of clathrin adaptors to signals on the mannose-6-phosphate/insulin-like growth factor II receptor. EMBO J. 8, 1041-1047.

Griffith, K. J., Chan, E. K. L., Lung, C. C., Hamel, J. C., Guo, X. Y., Miyachi, K., et al. (1997). Molecular cloning of a novel 97-kd Golgi complex autoantigen associated with Sjöogren's syndrome. Arthritis Rheum. 40, 1693-1702. doi: 10.1002/art.1780400920

Guo, Y., Punj, V., Sengupta, D., and Linstedt, A. D. (2008). Coat-tether interaction in Golgi organization. Mol. Biol. Cell 19, 2830-2843. doi: 10.1091/mbc.E07-121236

Hayes, G. L., Brown, F. C., Haas, A. K., Nottingham, R. M., Barr, F. A., and Pfeffer, S. R. (2009). Multiple Rab GTPase binding sites in GCC185 suggest a model for vesicle tethering at the trans-Golgi. Mol. Biol. Cell 20, 209-217. doi: 10.1091/mbc.E08-07-0740

Hoogenraad, C. C., Akhmanova, A., Howell, S. A., Dortland, B. R., De Zeeuw, C. I., Willemsen, R., et al. (2001). Mammalian Golgi-associated Bicaudal-D2 functions in the dynein-dynactin pathway by interacting with these complexes. EMBO J. 20, 4041-4054. doi: 10.1093/emboj/20.15. 4041

Houghton, F. J., Chew, P. L., Lodeho, S., Goud, B., and Gleeson, P. A. (2009). The localization of the Golgin GCC185 is independent of Rab6A/A' and Arl1. Cell 138, 787-794. doi: 10.1016/j.cell.2009.05.048

Ishida, R., Yamamoto, A., Nakayama, K., Sohda, M., Misumi, Y., Yasunaga, T., et al. (2015). GM130 is a parallel tetramer with a flexible rod-like structure and Nterminally open (Y-shaped) and closed (I-shaped) conformations. FEBS J. 282, 2232-2244. doi: 10.1111/febs.13271

Jing, J., Junutula, J. R., Wu, C., Burden, J., Matern, H., Peden, A. A., et al. (2010). FIP1/RCP binding to Golgin-97 regulates retrograde transport from recycling endosomes to the trans-Golgi network. Mol. Biol. Cell 21, 3041-3053. doi: 10.1091/mbc.E1004-0313

Kakinuma, T., Ichikawa, H., Tsukada, Y., Nakamura, T., and Toh, B. H. (2004). Interaction between p230 and MACF1 is associated with transport of a glycosyl phosphatidyl inositol-anchored protein from the Golgi to the cell periphery. Exp. Cell Res. 298, 388-398. doi: 10.1016/j.yexcr.2004. 04.047 
Kjer-Nielsen, L., Teasdale, R. D., van Vliet, C., and Gleeson, P. A. (1999). A novel Golgi-localisation domain shared by a class of coiled-coil peripheral membrane proteins. Curr. Biol. 9, 385-388. doi: 10.1016/S0960-9822(99) 80168-7

Lee, P. L., Ohlson, M. B., and Pfeffer, S. R. (2015). Rab6 regulation of the kinesin family KIF1C motor domain contributes to Golgi tethering. eLife 4:e06029. doi: 10.7554/eLife.06029

Lieu, Z. Z., and Gleeson, P. A. (2010). Identification of different itineraries and retromer components for endosome-to-Golgi transport of TGN38 and Shiga toxin. Eur. J. Cell Biol. 89, 379-393. doi: 10.1016/j.ejcb.2009.10.021

Lin, Y. C., Chiang, T. C., Liu, Y. T., Tsai, Y. T., Jang, L. T., and Lee, F. J. (2011). ARL4A acts with GCC185 to modulate Golgi complex organization. J. Cell Sci. 124, 4014-4026. doi: 10.1242/jcs.086892

Lu, L., and Hong, W. (2003). Interaction of Arl1-GTP with GRIP domains recruits autoantigens Golgin-97 and Golgin-245/p230 onto the Golgi. Mol. Biol. Cell 14, 3767-3781. doi: 10.1091/mbc.E03-01-0864

Lu, L., Tai, G., Wu, M., Song, H., and Hong, W. (2006). Multilayer interactions determine the Golgi localization of GRIP Golgins. Traffic 7, 1399-1407. doi: 10.1111/j.1600-0854.2006.00473.x

Luke, M. R., Kjer-Nielsen, L., Brown, D. L., Stow, J. L., and Gleeson, P. A. (2003). GRIP domain-mediated targeting of two new coiled-coil proteins, GCC88 and GCC185, to subcompartments of the trans-Golgi network. J. Biol. Chem. 278, 4216-4226. doi: 10.1074/jbc.M210387200

Malsam, J., Satoh, A., Pelletier, L., and Warren, G. (2005). Golgin tethers define subpopulations of COPI vesicles. Science 307, 1095-1108. doi: 10.1126/science. 1108061

Matanis, T., Akhmanova, A., Wulf, P., Del Nery, E., Weide, T., Stepanova, T., et al. (2002). Bicaudal-D regulates COPI-independent Golgi-ER transport by recruiting the dynein- dynactin motor complex. Nat. Cell Biol. 4, 986-992. doi: $10.1038 /$ ncb891

Matsui, T., Watanabe, T., Matsuzawa, K., Kakeno, M., Okumura, N., Sugiyama, I., et al. (2015). PAR3 and aPKC regulate Golgi organization through CLASP2 phosphorylation to generate cell polarity. Mol. Biol. Cell 26, 751-761. doi: 10.1091/mbc.E14-09-1382

Melancon, P., Glick, B. S., Malhotra, V., Weidman, P. J., Serafini, T., Gleason, M. L., et al. (1987). Involvement of GTP-binding "G" proteins in transport through the Golgi stack. Cell 51, 1053-1062. doi: 10.1016/0092-8674(87) 90591-5

Meyer, C., Zizioli, D., Lausmann, S., Eskelinen, E. L., Hamann, J., Saftig, P., et al. (2000). mulA-adaptin-deficient mice: lethality, loss of AP-1 binding and rerouting of mannose 6-phosphate receptors. EMBO J. 19, 2193-2203. doi: $10.1093 / \mathrm{emboj} / 19.10 .2193$

Munro, S. (2011). The Golgin coiled-coil proteins of the Golgi apparatus. Cold Spring Harb. Perspect. Biol. 3:a005256. doi: 10.1101/cshperspect.a0 05256

Munro, S., and Nichols, B. J. (1999). The GRIP domain- a novel Golgi- targeting domain found in several coiled-coil proteins. Curr. Biol. 9, 377-380. doi: 10.1016/S0960-9822(99)80166-3

Orci, L., Perrelet, A., and Rothman, J. E. (1998). Vesicles on strings: morphological evidence for processive transport within the golgi stack. Proc. Natl. Acad. Sci. U.S.A. 95, 2279-2283. doi: 10.1073/pnas.95.5.2279

Panic, B., Perisic, O., Veprintsev, D. B., Williams, R. L., and Munro, S. (2003). Structural basis for Arl1-dependent targeting of homodimeric GRIP domains to the Golgi apparatus. Mol. Cell 12, 863-874. doi: 10.1016/S10972765(03)00356-3

Pei, J., Kim, B., and Grishin, N. V. (2008). PROMALS3D: a tool for multiple sequence and structure alignment. Nucl. Acids Res. 36, 2295-2300. doi: 10.1093/nar/gkn072

Pfeffer, S. R. (1999). Transport-vesicle targeting: tethers before SNAREs. Nat. Cell Biol. 1, E17-E22. doi: 10.1038/8967

Pranke, I. M., Morello, V., Bigay, J., Gibson, K., Verbavatz, J. M., Antonny, B., et al. (2011). $\alpha$-Synuclein and ALPS motifs are membrane curva- ture sensors whose contrasting chemistry mediates selective vesicle binding. J. Cell Biol. 194, 89-103. doi: 10.1083/jcb.201011118

Puthenveedu, M. A., Bachert, C., Puri, S., Lanni, F., and Linstedt, A. D. (2006). GM130 and GRASP65-dependent lateral cisternal fusion confers uniform Golgi enzyme distribution. Nat. Cell Biol. 8, 238-248. doi: 10.1038/ncb1366
Puthenveedu, M. A., and Linstedt, A. D. (2004). Gene replacement reveals that p115/SNARE interactions are essential for Golgi biogenesis. Proc. Natl. Acad. Sci. U.S.A. 101, 1253-1256. doi: 10.1073/pnas.0306373101

Ramirez, I. B., and Lowe, M. (2009). Golgins and GRASPs: holding the Golgi together. Semin. Cell Dev. Biol. 20, 770-779. doi: 10.1016/j.semcdb.2009.03.011

Reddy, J. V., Burguete, A. S., Sridevi, K., Ganley, I. G., Nottingham, R. M., and Pfeffer, S. R. (2006). A functional role for the GCC185 Golgin in mannose 6-phosphate receptor recycling. Mol. Biol. Cell 17, 4353-4363. doi: 10.1091/mbc.E06-02-0153

Robert, X., and Gouet, P. (2014). Deciphering key features in protein structures with the new ENDscript server. Nucl. Acids Res. 42, 320-324. doi: 10.1093/nar/gku316

Sapperstein, S. K., Walter, D. M., Grosvenor, A. R., Heuser, J. E., and Waters, M. G. (1995). p115 is a general vesicular transport factor related to the yeast ER to Golgi transport factor Usolp. Proc. Natl. Acad. Sci. U.S.A. 92, 522-526. doi: 10.1073/pnas.92.2.522

Sato, K., Roboti, P., Mironov, A. A., and Lowe, M. (2015). Coupling of vesicle tethering and Rab binding is required for in vivo functionality of the Golgin GMAP-210. Mol. Biol. Cell 26, 537-553. doi: 10.1091/mbc.E14-10-1450

Schlager, M. A., Kapitein, L. C., Grigoriev, I., Burzynski, G. M., Wulf, P. S., Keijzer, N., et al. (2010). Pericentrosomal targeting of Rab6 secretory vesicles by Bicaudal-D-related protein 1 (BICDR-1) regulates neuritogenesis. EMBO J. 29, 1637-1651. doi: 10.1038/emboj.2010.51

Short, B., Haas, A., and Barr, F. A. (2005). Golgins and GTPases, giving identity and structure to the Golgi apparatus. Biochim. Biophys. Acta 1744, 383-395. doi: 10.1016/j.bbamcr.2005.02.001

Short, B., Preisinger, C., Schaletzky, J., Kopajtich, R., and Barr, F. A. (2002). The Rab6 GTPase regulates recruitment of the dynactin complex to Golgi membranes. Curr. Biol. 12, 1792-1795. doi: 10.1016/S0960-9822(02) 01221-6

Shorter, J., Beard, M. B., Seemann, J., Dirac-Svejstrup, A. B., and Warren, G. (2002). Sequential tethering of Golgins and catalysis of SNAREpin assembly by the vesicle-tethering protein p115. J. Cell Biol. 157, 45-62. doi: $10.1083 /$ jcb. 200112127

Simon, J.-P., Ivanov, I. E., Adesnik, M., and Sabatini, D. D. (1996). The production of post Golgi vesicles requires a protein kinase C-like molecule, but not its phosphorylating activity. J. Cell Biol. 135, 335-370. doi: 10.1083/jcb. 135.2.355

Siniossoglou, S. (2005). Affinity purification of Ypt6 effectors and identification of TMF/ARA160 as a Rab6 interactor. Meth. Enzymol. 403, 599-607. doi: 10.1016/S0076-6879(05)03052-1

Siniossoglou, S., and Pelham, H. R. (2001). An effector of Ypt6p binds the SNARE Tlg1p and mediates selective fusion of vesicles with late Golgi membranes. EMBO J. 20, 5991-5998. doi: 10.1093/emboj/20.21.5991

Sinka, R., Gillingham, A. K., Kondylis, V., and Munro, S. (2008). Golgi coiled-coil proteins contain multiple binding sites for Rab family $\mathrm{G}$ proteins. J. Cell Biol. 183, 607-615. doi: 10.1083/jcb.200808018

Torres, I. L., Rosa-Ferreira, C., and Munro, S. (2014). The Arf family G protein Arl1 is required for secretory granule biogenesis in Drosophila. J. Cell Sci. 127, 2151-2160. doi: 10.1242/jcs. 122028

van Valkenburgh, H., Shern, J. F., Sharer, J. D., Zhu, X., and Kahn, R. A. (2001). ADP-ribosylation factors (ARFs) and ARF-like 1 (ARL1) have both specific and shared effectors: characterizing ARL1-binding proteins. J. Biol. Chem. 276, 22826-22837. doi: 10.1074/jbc.M102359200

Weidman, P., Roth, R., and Heuser, J. (1993). Golgi membrane dynamics imaged by freeze-etch electron microscopy: views of different membrane coatings involved in tubulation versus vesiculation. Cell 75, 123-133. doi: 10.1016/S0092-8674(05)80089-3

Wong, M., and Munro, S. (2014). Membrane trafficking. The specificity of vesicle traffic to the Golgi is encoded in the Golgin coiled-coil proteins. Science 346:1256898. doi: 10.1126/science. 1256898

Wu, M., Lu, L., Hong, W., and Song, H. (2004). Structural basis for recruitment of GRIP domain Golgin-245 by small GTPase Arl1. Nat. Struct. Mol. Biol. 11, 1-10. doi: 10.1038/nsmb714

Yamakawa, H., Seog, D. H., Yoda, K., Yamasaki, M., and Wakabayashi, T. (1996). Usol protein is a dimer with two globular heads and a long coiled-coil tail. J. Struct. Biol. 116, 356-365. doi: 10.1006/jsbi.1996.0053 
Yamane, J., Kubo, A., Nakayama, K., Yuba-Kubo, A., Katsuno, T., and Tsukita, S., Tsukita, S. (2007). Functional involvement of TMF/ARA160 in Rab6dependent retrograde membrane traffic. Exp. Cell Res. 313, 3472-3485. doi: 10.1016/j.yexcr.2007.07.010

Yoshino, A., Setty, S. R., Poynton, C., Whiteman, E. L., Saint-Pol, A., Burd, C. G., et al. (2005). tGolgin-1 (p230, golgin-245) modulates Shiga-toxin transport to the Golgi and Golgi motility towards the microtubule-organizing centre. J. Cell Sci. 118, 2279-2293. doi: 10.1242/jcs.02358

Zolov, S. N., and Lupashin, V. V. (2005). Cog3p depletion blocks vesicle-mediated Golgi retrograde trafficking in HeLa cells. J. Cell Biol. 168, 747-759. doi: $10.1083 /$ jcb. 200412003
Conflict of Interest Statement: The authors declare that the research was conducted in the absence of any commercial or financial relationships that could be construed as a potential conflict of interest.

Copyright (C) 2016 Cheung and Pfeffer. This is an open-access article distributed under the terms of the Creative Commons Attribution License (CC BY). The use, distribution or reproduction in other forums is permitted, provided the original author(s) or licensor are credited and that the original publication in this journal is cited, in accordance with accepted academic practice. No use, distribution or reproduction is permitted which does not comply with these terms. 